\title{
Bone Flap Changes after Cranioplasty Using Frozen Autologous Bone Flaps: A Three-Dimensional Volumetric Reconstruction Study
}

\author{
Jung Hwan Lee ${ }^{1}$, Chung Kee Chough ${ }^{2}$, Hyuk Jin Choi ${ }^{1}$, Jun Kyeung Ko ${ }^{1}$, \\ Won Ho $\mathrm{Cho}^{1}$, Seung Heon Cha ${ }^{1}$, Chang Hwa Choi ${ }^{1}$, and Young Ha Kim ${ }^{3}$ \\ ${ }^{1}$ Department of Neurosurgery, Pusan National University Hospital, Busan; \\ ${ }^{2}$ Catholic Neuroscience Center, Department of Neurosurgery, St. Mary's Hospital, College of Medicine, The Catholic University of Korea, Seoul; \\ ${ }^{3}$ Department of Neurosurgery, Pusan National University Yangsan Hospital, Yangsan, Korea.
}

Purpose: Bone flap resorption (BFR) after cranioplasty with an autologous bone flap (ABF) is well known. However, the prevalences and degrees of BFR remain unclear. This study aimed to evaluate changes in ABFs following cranioplasty and to investigate factors related with BFR.

Materials and Methods: We retrospectively reviewed 97 patients who underwent cranioplasty with frozen ABF between January 2007 and December 2016. Brain CT images of these patients were reconstructed to form three-dimensional (3D) images, and 3D images of $\mathrm{ABF}$ were separated using medical image editing software. ABF volumes on images were measured using 3D image editing software and were compared between images in the immediate postoperative period and at postoperative 12 months. Risk factors related with BFR were also analyzed.

Results: The volumes of bone flaps calculated from CT images immediately after cranioplasty ranged from $55.3 \mathrm{~cm}^{3}$ to $175 \mathrm{~cm}^{3}$. Remnant bone flap volumes at postoperative 12 months ranged from $14.2 \%$ to $102.5 \%$ of the original volume. Seventy-five patients (77.3\%) had a BFR rate exceeding $10 \%$ at 12 months after cranioplasty, and 26 patients (26.8\%) presented severe BFR over $40 \%$. Ten patients (10.3\%) underwent repeated cranioplasty due to severe BFR. The use of a 5-mm burr for central tack-up sutures was significantly associated with BFR $(p<0.001)$.

Conclusion: Most ABFs after cranioplasty are absorbed. Thus, when using frozen ABF, patients should be adequately informed. To prevent $\mathrm{BFR}$, making holes must be kept to a minimum during $\mathrm{ABF}$ grafting.

Key Words: Autografts, bone resorption, cranioplasty, decompressive craniectomy, skull

\section{INTRODUCTION}

Many studies have demonstrated that decompressive craniec-

Received: April 16, 2019 Revised: August 14, 2019

Accepted: August 20, 2019

Corresponding author: Chung Kee Chough, MD, PhD, Catholic Neuroscience Center, Department of Neurosurgery, St. Mary's Hospital, College of Medicine, The Catholic University of Korea, 63ro 10, Yeongdeungpo-gu, Seoul 07345, Korea. Tel: 82-2-3779-1187, Fax: 82-2-786-5809, E-mail: chough@catholic.ac.kr

-The authors have no potential conflicts of interest to disclose.

(C) Copyright: Yonsei University College of Medicine 2019

This is an Open Access article distributed under the terms of the Creative Commons Attribution Non-Commercial License (https://creativecommons.org/licenses/ by-nc/4.0) which permits unrestricted non-commercial use, distribution, and reproduction in any medium, provided the original work is properly cited. tomy is effective in reducing intracranial pressure in cases of severe traumatic brain injury, severe cerebral infarction, and excessive spontaneous intracranial hemorrhage.$^{1-3}$ However, once the cerebral edema has subsided, additional surgical treatment, a cranioplasty, is needed. ${ }^{4}$ Generally, cranioplasty after decompressive craniectomy is recommended for the following reasons: First, it can improve neurologic function by reestablishing cerebrospinal fluid dynamics and cerebral blood flow. ${ }^{5,6}$ Second, it protects the brain from physical impact. ${ }^{7}$ Finally, it enables cosmetic restoration of the cranial contour. ${ }^{4}$

For decades, the most commonly used material in cranioplasty was deeply frozen autologous bone flap (ABF) ${ }^{4,8}$ However, some studies have reported that $\mathrm{ABF}$ is associated with significant complications, such as bone flap resorption (BFR) ${ }^{49,10}$ While 
minimal BFR may not lead to functional complications, it may cause appearance problems depending on the resorption site. Severe BFR not only causes a cosmetic problem but also diminishes brain protection function. Thus, the advantages of cranioplasty are eliminated, ${ }^{11}$ and an additional cranioplasty procedure may be required. ${ }^{10}$

However, there is no consensus on the definition of BFR or criteria for the severity thereof. ${ }^{4}$ In addition, few studies have investigated changes in bone flap volume following cranioplasty with frozen $\mathrm{ABF}$. Currently, CT has significantly advanced to represent the three-dimensional (3D) structures of the living human body. ${ }^{12}$ In addition, medical image editing and computer-aided design (CAD) software have recently been improved and become easy to use. ${ }^{12}$ Using medical CAD software, we are able to separate $\mathrm{ABF}$ regions from the whole skull on CT scans. The separated $\mathrm{ABF}$ can be reconstructed to $3 \mathrm{D}$ images that can be further processed for volume measurement and identification of shape changes. The aim of this study was to evaluate changes in $\mathrm{ABF}$ volume following cranioplasty using medical CAD software and to analyze the incidence, severity, pattern, and risk factors of BFR.

\section{MATERIALS AND METHODS}

\section{Patient population}

From January 2007 to December 2016, 394 patients underwent 404 cranioplasty surgical procedures following decompressive craniectomy. Of these patients, those with a follow-up period of less than 12 months and those in whom an artificial implant (bone cement or titanium mesh) was used were excluded. Hence, we retrospectively reviewed 97 patients who underwent cranioplasty using frozen ABF (Fig. 1).

\section{Surgical technique}

Decompressive craniectomy was performed according to the standard technique of frontotemporoparietal craniectomy fol-

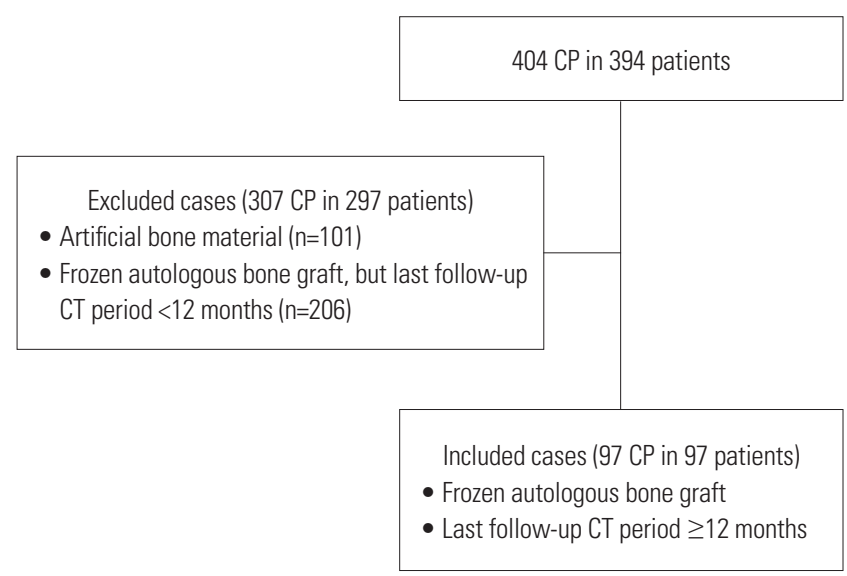

Fig. 1. Flowchart of cases included in or excluded from the study. CP, cranioplasty. lowed by duroplasty. The dura mater was incised in the shape of an asterisk. After the durotomy, the exposed brain was covered with an artificial graft, and watertight duroplasty was not performed. The artificial graft was derived from bovine pericardium. After craniectomy, ABF was stored according to the following procedure: the soft tissues attached to $\mathrm{ABF}$ were completely removed, and the ABF was irrigated with normal saline supplemented with antibiotics and placed in a sterile plastic bag. ${ }^{13,14}$ Subsequently, ABF was sealed in at least three bags. ${ }^{14-16}$ Afterwards, the sealed ABF was placed in a deep freezer at a temperature of $-70^{\circ} \mathrm{C}^{14-16}$

After decompressive craniectomy, cranioplasty was performed as soon as possible if scalp depression was identified according to decreased intracranial pressure. However, in cases of highrisk general anesthesia, such as myocardial infarction, unstable angina, or pneumonia, delayed cranioplasty was recommended. Cranioplasty was performed as follows: Before surgery, the frozen $\mathrm{ABF}$ was thawed in sterile warm saline supplemented with antibiotics. ${ }^{14}$ A skin incision was made at the previous incision site. The temporalis muscle and the scalp were separated from the dura mater so that the ABF could be anatomically repositioned. In cases where the superficial temporal artery had been preserved during the previous craniectomy surgery, we tried to identify and preserve it. The periosteum and granulation tissue of the bone margin were removed. ABF was drilled, and small holes for central dura tenting suture were made. ABF was repositioned and fixed with titanium miniplates. The ABF was attached to the forehead as closely as possible to prevent the formation of a bony gap at the forehead. After central tenting suturing, a drain was inserted under the temporalis muscle, and the skin was closed.

\section{Clinical data}

Upon approval of our study by the Institutional Review Board of Pusan National University Hospital (IRB No. 1810-018-072), we collected the CT images obtained immediately after cranioplasty and at postoperative 12 months. In patients whose CT scans were performed consequently at postoperative 3 months, 6 months, and 12 months, CT images at postoperative 3 months and 6 months were also collected. In patients whose CT scans were performed after 12 months, the last follow-up CT images were collected. All CT scans were taken helically. The peak kilovoltage of CT scans was 120, and the slice thickness of CT scans was $1 \mathrm{~mm}$. Tube currents were set automatically by each CT machine and ranged from $160 \mathrm{~mA}$ to $420 \mathrm{~mA}$. All CT images from the same patient were taken on the same CT machine. CT images were collected in the Digital Imaging and Communications in Medicine (DICOM) file format. The following medical records were reviewed for patients-related factors relevant to bone resorption: age, sex, past medical history, initial Glasgow Coma Scale score, Glasgow Outcome Scale at postoperative 12 months, the reason for craniectomy, and shunt-dependent hydrocephalus. There was no overlapping in past medical history. Further, 
we reviewed surgeon-related factors, such as institutions to which each certified neurosurgeon belong, initial bone volume, preservation of the superficial temporal artery, duration from craniectomy to cranioplasty, operation time of cranioplasty, and making wide holes using a 5-mm drill burr for central tack-up suturing. In addition, cases of repeated cranioplasty due to severe BFR were collected and reviewed.

\section{D modeling and volume calculation of $\mathrm{ABF}$}

To measure the volume of ABF, we used Materialise Mimics version 20 (Materialise Co., Leuven, Belgium) to edit DICOM files and convert them to the stereolithography (STL) file format. In detail, after opening Materialise Mimics, we imported the DICOM files and ran the "thresholding" function by selecting the "bone" option. This automatically selected an area between 226 and 3071 Hounsfield units on all DICOM files. When the mask corresponding to the bone was created, we selected the function "Calculate 3D" to 3D model the whole skull from the CT scans. Subsequently, we manually selected only the ABF region using the "edit mask" option (Fig. 2). The edited mask was reconstructed in 3D using the "Calculate 3D" function and was converted into the STL format. We imported the STL file of each ABF into Autodesk Netfabb version 2017 (Autodesk Co., San Rafael, CA, USA) and calculated the volume of ABF. The volume of the remnant bone flap during the final follow-up was calculated as the percentile of the initial bone flap volume.

\section{Definition of bone flap preservation and resorption}

We divided the patients into two groups to identify factors related with bone resorption. The bone flap preservation (BFP) group comprised those in whom the volume of the remnant bone flap was more than $90 \%$ of the initial bone flap volume. Patients in whom the volume of remnant bone flap was less than $90 \%$ of the initial bone volume were classified into the BFR group. Cas-
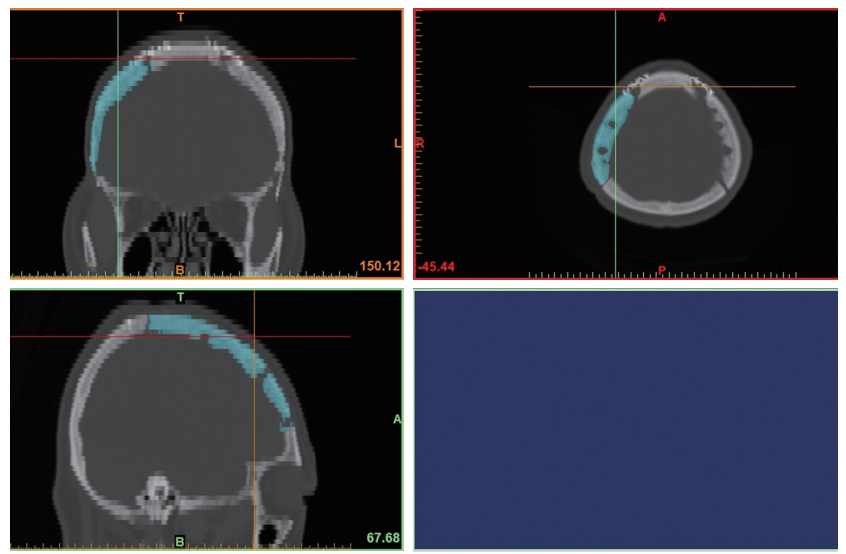

Fig. 2. Volume measurement with 3D modeling software. Using Materialise Mimics, we selected autologous bone flaps from CT scans of the skull and reconstructed them into $3 \mathrm{D}$ images. The reconstructed images were converted into STL files for 3D images. The STL file of the bone flap was imported by Netfabb to calculate the volume. 3D, three dimensional; STL, stereolithography. es in which the volume of the remnant bone flap was less than $60 \%$ of the initial volume were classified as exhibiting "severe BFR." In addition, we divided the patients into those who underwent repeated cranioplasty due to bone resorption and those who did not.

\section{Statistical analysis}

All statistical analyses were conducted using SPSS version 18.0 (SPSS Inc., Chicago, IL, USA). Categorical data were analyzed using chi-square test. If the expected frequency of categorical data was lower than five, Fisher's exact test was used. Continuous variables with a sample size of 30 or more were tested by Student's t-test. If the sample size was lower than 30 and the normality was not verified, Mann-Whitney U test was used. Probability values of less than 0.05 were considered to be statistically significant.

\section{RESULTS}

The characteristics of the study are summarized in Table 1 . The mean actual volume of initial bone flaps was $83.72 \mathrm{~cm}^{3}$ (ranging from $55.3 \mathrm{~cm}^{3}$ to $175 \mathrm{~cm}^{3}$ ). The mean actual volume of the bone flaps on CT scans at 12 months after surgery was $58.52 \mathrm{~cm}^{3}$ (ranging from $10.8 \mathrm{~cm}^{3}$ to $121.9 \mathrm{~cm}^{3}$ ). The mean volume of the remnant bone flap at 12 months after surgery was $73.4 \%$ of the initial bone flap volume (ranging from $14.2 \%$ to $102.5 \%$ ). Considering the final follow-up brain CT scans, 75 patients were included in the BFR group (77.3\%). Among them, there were 26 (26.8\%) severe BFR cases. In the BFR group, the mean volume of remnant bone flaps at 12 months after surgery was $66.5 \%$ of the initial bone flap volume. Twenty-two patients were classified in the

Table 1. Characteristics of the Patients Who Underwent Autologous Cranioplasty with a Follow-Up Period of 12 Months or Longer ( $n=97$ )

\begin{tabular}{lc}
\hline \multicolumn{1}{c}{ Variable } & Value \\
\hline Age (yr) & $47.0 \pm 16.2$ \\
Male:female & $71: 26$ \\
Follow-up period after cranioplasty (month) & $24.70 \pm 17.86$ \\
GCS score at craniectomy & $8.40 \pm 2.95$ \\
GOS score at 12 months & $3.8 \pm 1.0$ \\
Underlying disease & \\
\hline Diabetes mellitus & $8(8.2)$ \\
\hline Hypertension & $12(12.4)$ \\
\hline Arterial fibrillation & $3(3)$ \\
Reason of craniectomy & \\
\hline Trauma & $71(73.2)$ \\
\hline Cerebral infarction & $8(8.2)$ \\
\hline Spontaneous intracranial hemorrhage & $18(18.6)$ \\
\hline Duration from craniectomy to cranioplasty (month) & $3.34 \pm 2.25$ \\
Repeated cranioplasty cases due to bone resorption & $10(10.3)$ \\
\hline
\end{tabular}

GCS, Glasgow Coma Scale; GOS, Glasgow Outcome Scale.

Data are presented as mean \pm SD or number (\%). 
BFP group, and the mean volume of the remnant bone flap was $97.1 \%$ of the initial bone flap volume. The distribution of remnant bone flaps at 12 months postoperatively is demonstrated in Fig. 3.

In 13 patients from the BFR group, CT scans were consecutively obtained at 3, 6, and 12 months. In these patients, the mean volume of the remnant bone flap at 3 months was $81.6 \%$ of the initial bone flap volume. At 6 months, the mean volume was $64.1 \%$ of the initial bone flap volume and had decreased to $52.1 \%$ at 12 months (Fig. 4). Among these patients, the parietal bone was the most common site at which BFR was initiated $(n=12)$. The next most frequent sites for the initiation of BFR were surgically-made holes $(n=11)$ and temporal bone $(n=10)$. Notably, most cases of BFR started at two or three places at the same time. In fewer cases, BFR occurred in the frontal bone $(n=4)$ (Fig. 5). In 25 patients, the follow-up period was longer than 12 months (24 to 96 months). In these patients, at 12 months,

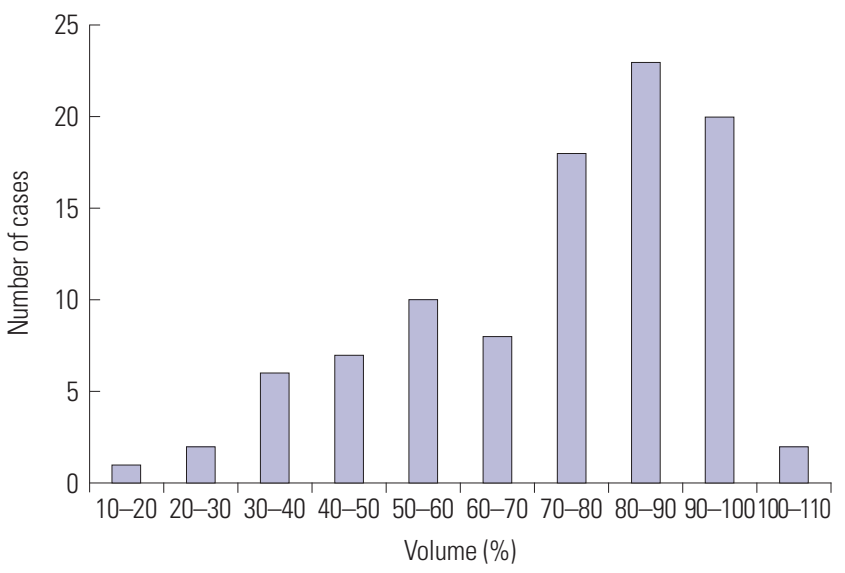

Fig. 3. Distribution of volumes of remnant bone flap.

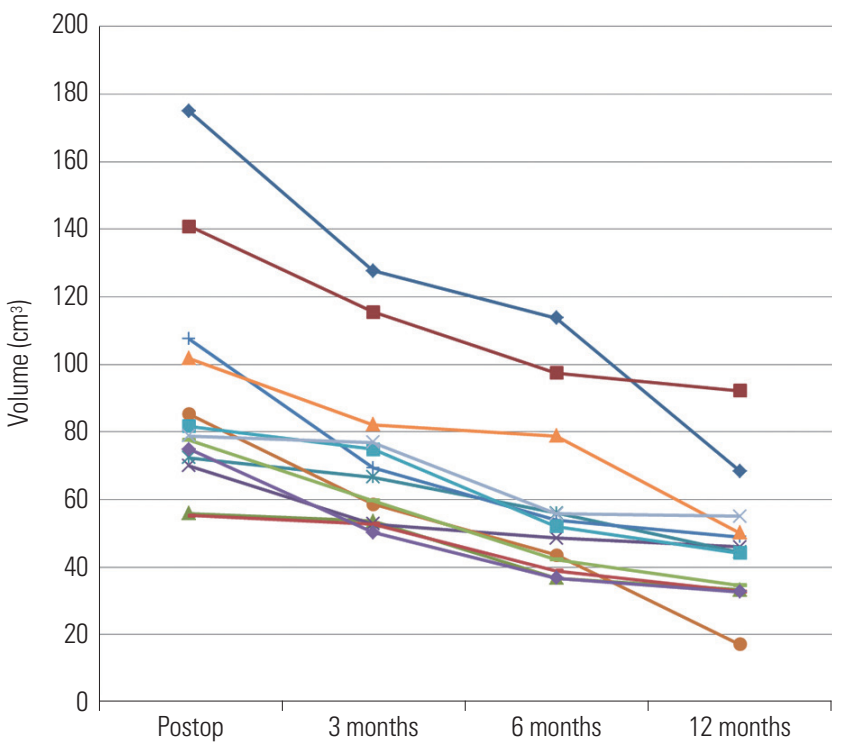

Fig. 4. Changes in volume of $A B F$ of 13 patients with serial computed tomography scans at 3,6 , and 12 months in the bone flap resorption group. On average, the volume of ABF at 12 months was preserved by an average of $52.1 \%$. $A B F$, autologous bone flap. the mean volume was $70.3 \%$ of the initial bone flap. At final follow up, the mean volume of remnant bone flaps was $64.5 \%$. Thus, BFR had progressed further after 12 months $(p<0.001)$. However, the progression severity of BFR after 12 months was lower than that observed during the first 12 months $(29.7 \%$ vs. $5.8 \%, p<0.001$ ) (Fig. 6).

When comparing the BFP group with the BFR group, no patient-related factors were significantly associated with BFR (Table 2). Among surgeon-related factors, making large holes using a 5-mm burr was significantly associated with BFR $(p<0.001)$ (Table 3).

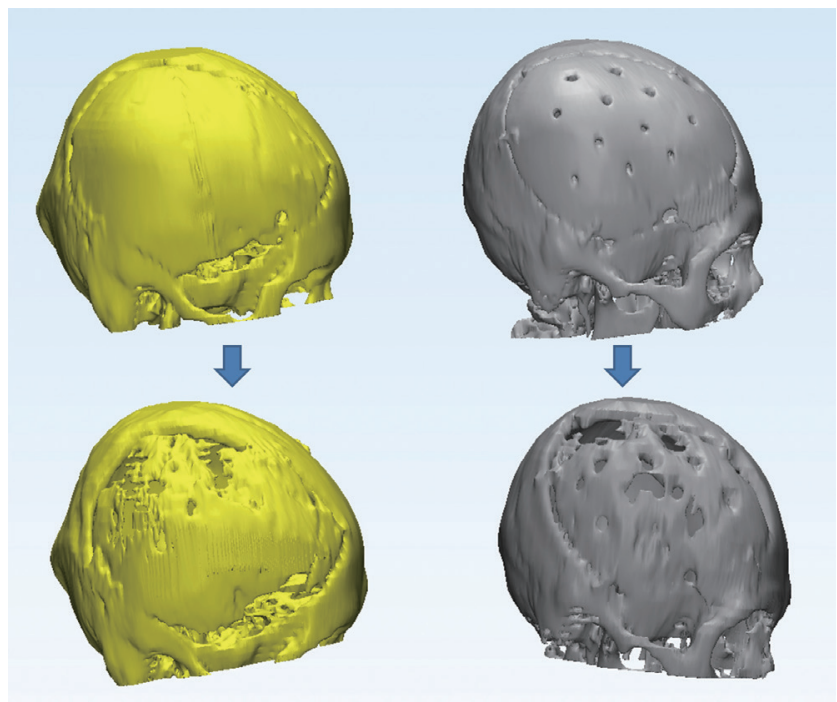

Fig. 5. Changes in the skull and ABF after cranioplasty in the BFR group. In general, BFR began in the parietal bone and the temporal bone (left). If wide holes existed on $A B F$, BFR was also initiated at the holes (right). ABF, autologous bone flap; BFR, bone flap resorption.

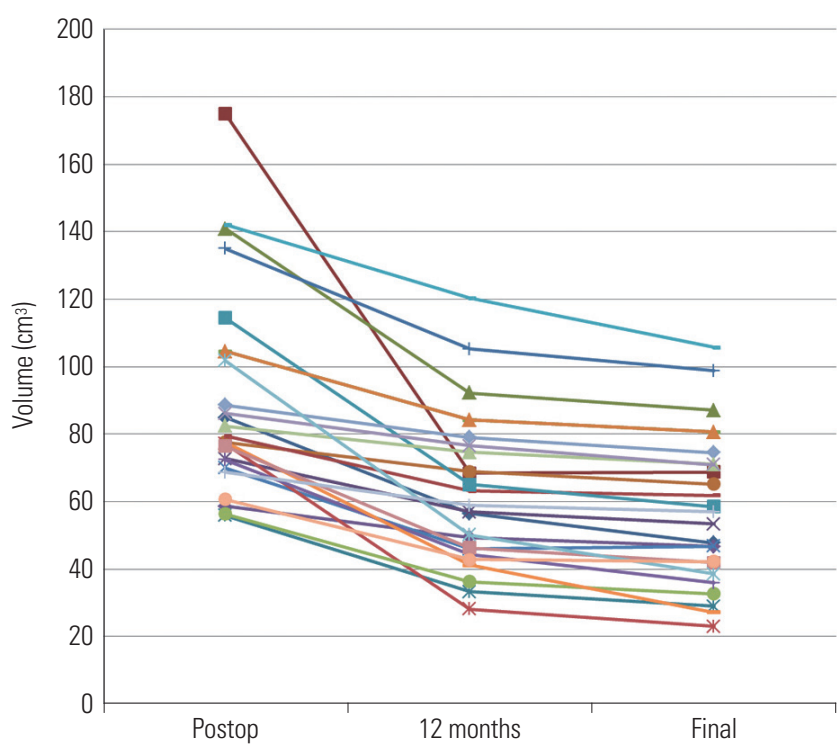

Fig. 6. Changes in the volume of $A B F$ after 12 months in the bone flap resorption group. Unlike before 12 months, the decline decreased after 12 months. After an additional 12 months, the volume of ABF decreased only by an average of $5.8 \%$. ABF, autologous bone flap. 
Ten patients underwent repeated cranioplasty due to significant BFR. All of these patients complained about their appearance following BFR. The minimum follow-up period was 12 months in these patients, and there was no patient who underwent repeated cranioplasty due to significant BFR before this follow-up period. These patients are listed and described in Table 4 . Of the 26 patients with severe BFR, 17 did not undergo reoperation. In six of these patients, neurosurgeons recognized significant BFR and recommended reoperation, but the patients refused. In the remaining 11 patients with severe BFR, there was

Table 2. Univariate Analysis of Patient-Related Factors between the Bone Flap Preservation and Resorption Groups

\begin{tabular}{|c|c|c|c|}
\hline Factors & Preservation group ( $\mathrm{n}=22$ ) & Resorption group ( $\mathrm{n}=75$ ) & $p$ value \\
\hline Age (yr) & $50.50 \pm 13.94$ & $45.91 \pm 16.70$ & 0.243 \\
\hline Age group (under 30 years-old) (yes:no) & $3: 19$ & $16: 59$ & 0.550 \\
\hline GCS score at craniectomy & $8.270 \pm 2.881$ & $8.390 \pm 2.981$ & 0.874 \\
\hline GOS score at 12 months & $4.050 \pm 0.999$ & $3.770 \pm 1.008$ & 0.267 \\
\hline Sex (male:female) & $2: 1$ & $3.4: 1$ & 0.589 \\
\hline Hydrocephalus requiring shunting (yes:no) & $6: 16$ & 18:57 & 0.782 \\
\hline Past history & & & 0.345 \\
\hline None & 17 & 57 & \\
\hline Diabetes mellitus & 0 & 8 & \\
\hline Hypertension & 4 & 8 & \\
\hline Arterial fibrillation & 1 & 2 & \\
\hline Cause of craniectomy & & & 0.444 \\
\hline Trauma & 14 & 57 & \\
\hline Cerebral infarction & 3 & 5 & \\
\hline Spontaneous intracranial hemorrhage & 5 & 13 & \\
\hline
\end{tabular}

GCS, Glasgow Coma Scale; GOS, Glasgow Outcome Scale.

Data are presented as mean $\pm S D$ or number.

Table 3. Univariate Analysis of Surgeon-Related Factors between the Bone Flap Preservation and Resorption Groups

\begin{tabular}{lccc}
\hline \multicolumn{1}{c}{ Factors } & Preservation group (n=22) & Resorption group (n=75) & p value \\
\hline Superficial temporal artery preservation (yes:no) & $8: 14$ & $22: 53$ & 0.602 \\
Initial bone flap volume & $86.9 \pm 28.0$ & $82.8 \pm 28.0$ & 0.547 \\
Duration from craniectomy to cranioplasty & $3.14 \pm 2.60$ & $3.40 \pm 2.15$ & 0.631 \\
Operation time of cranioplasty & $124.0 \pm 34.7$ & $128.0 \pm 34.8$ & 0.664 \\
Wide holes on bone flap using 5-mm burr (yes:no) & $2: 20$ & $40: 35$ & $<0.001$ \\
Institution & & & 0.134 \\
A & 4 & 43 & 15 \\
B & 9 & & \\
C & 9 & &
\end{tabular}

Data are presented as mean $\pm S D$ or number.

Table 4. Summary of Repeated Cranioplasty Cases Due to Significant Resorption of Autologous Bone Flap

\begin{tabular}{|c|c|c|c|c|c|c|c|c|}
\hline Cases & Sex & $\begin{array}{l}\text { Age } \\
\text { (yr) }\end{array}$ & Diagnosis & $\begin{array}{c}\text { Duration from craniectomy } \\
\text { to CP (month) }\end{array}$ & $\begin{array}{c}\text { Period of repeated } \\
\text { CP (month) }\end{array}$ & $\begin{array}{c}\text { Remnant flap volume } \\
\text { at } 12 \text { months }(\%)\end{array}$ & $\begin{array}{l}\text { Remnant flap volume } \\
\text { at repeated CP (\%) }\end{array}$ & $\begin{array}{c}\text { Initial bone flap } \\
\text { volume }\left(\mathrm{cm}^{3}\right)\end{array}$ \\
\hline 1 & $M$ & 55 & Trauma & 2 & 17 & 40.2 & 38.7 & 83.878 \\
\hline 2 & $M$ & 24 & Trauma & 1 & 14 & 58.1 & 56.1 & 84.945 \\
\hline 3 & M & 57 & Trauma & 2 & 24 & 40.1 & 31.7 & 63.219 \\
\hline 4 & $\mathrm{~F}$ & 60 & Trauma & 2 & 12 & 14.2 & 14.2 & 76.375 \\
\hline 5 & M & 58 & Trauma & 3 & 15 & 68.1 & 67.2 & 71.046 \\
\hline 6 & M & 14 & Trauma & 4 & 54 & 56.7 & 46.3 & 83.832 \\
\hline 7 & $\mathrm{~F}$ & 53 & $\mathrm{~S}-\mathrm{ICH}$ & 2 & 20 & 38.7 & 35.0 & 77.555 \\
\hline 8 & M & 23 & Trauma & 2 & 14 & 20.1 & 20.1 & 85.198 \\
\hline 9 & $\mathrm{M}$ & 60 & Trauma & 6 & 12 & 59.3 & 59.3 & 55.274 \\
\hline 10 & M & 23 & Trauma & 1 & 12 & 43.7 & 43.7 & 74.898 \\
\hline
\end{tabular}

CP, cranioplasty; S-ICH, spontaneous intracranial hemorrhage. 
no mention of BFR in the medical records.

\section{DISCUSSION}

Autologous bone resorption following cranioplasty has been well described. The incidence has been reported to range from $3 \%$ to $12 \%$ in adult patients and to reach $50 \%$ in pediatric patients. ${ }^{4,8}$ However, there is no precise definition for BFR. Moreover, there are several methods that can be used to measure the amount of the remaining bone flap; however, there is no standard protocol. ${ }^{4,10,17}$ The strength of our study was that we were able to calculate, with high precision, the actual volume of ABFs through CT images and 3D CAD software and to define with accuracy changes in the volume thereof over time after surgery.

In this study, we defined BFR as a higher than $10 \%$ decrease in the bone flap volume. Measuring the $\mathrm{ABF}$ volume in the reconstructed 3D image is rather accurate due to the unbiased calculations performed by the 3D image software. However, in the step of manually selecting the bone flap region in the CT images, some edges may have been selected slightly inaccurately. Thus, even when working with the same CT image, there is a possibility that the calculated volume is slightly different. Therefore, we did not define all cases with a volume decrease as BFR, and only considered cases with a change of at least of $10 \%$.

We defined severe BFR as a volume reduction of more than $40 \%$. This condition could be similar to bone resorption as defined in previous relevant studies. We determined this value with reference to repeated cranioplasty and recognition of BFR by neurosurgeons. In patients who underwent repeated cranioplasty, the largest volume of the remnant bone flap was estimated at $67 \%$ of its original volume (Fig. 7). Additionally, there were seven cases in which neurosurgeons recognized and recorded the occurrence of BFR even though they had not undergone repeated cranioplasty. In these cases, the largest volume of the remnant bone flap was $61 \%$ of its original volume. Patients, caregivers and neurosurgeons could recognize BFR if the volume of the remnant bone flap was less than $60 \%$ of its original volume. Thus, we defined this range as "severe BFR."

Several risk factors for BFR following cranioplasty have been suggested in previous studies. The suggested factors include young age, bone flap fragmentation, shunt-dependent hydrocephalus, large skull defect, and a longer period from craniectomy to cranioplasty. ${ }^{10,18,19}$ However, these risk factors were identified differently by different studies. For example, one study concluded that only a large skull defect is related with BFR, while the remaining factors are irrelevant. ${ }^{4}$ In another study, young age was identified as a risk factor, but no relation was reported between other factors and BFR. ${ }^{10}$ In this study, the factors mentioned above were not found to be associated with BFR. Recently, there have been some reports suggesting that BFR is more likely to occur in patients younger than 30 years. ${ }^{19}$ Therefore, in this paper, age groups were classified according to this age, al-

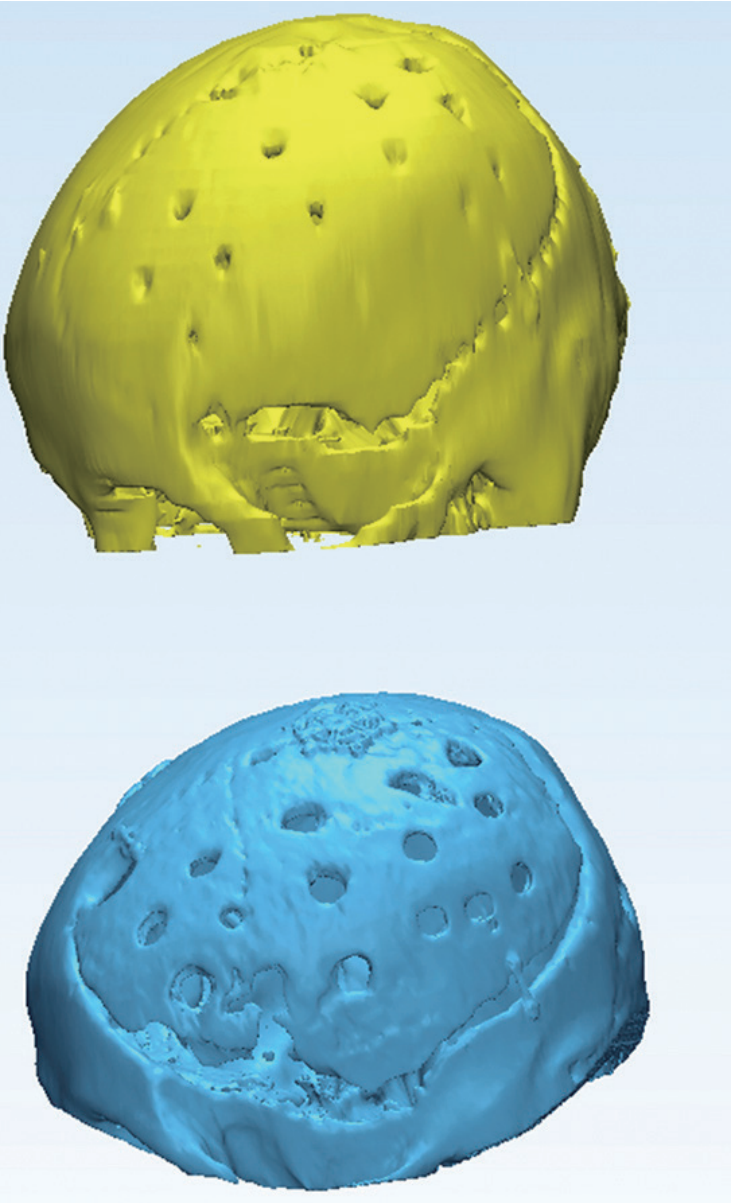

Fig. 7. Three-dimensional images of the cranium following cranioplasty. The upper image was obtained from immediate postoperative CT scans, and the lower image was obtained from CT scans at 12 months after surgery. The volume of the remaining autologous bone flap was $67 \%$ of its original volume, and repeated cranioplasty was performed due to appearance issues that caused depression in the forehead area.

though it was not related to the occurrence of BFR. Instead, making large holes in the $\mathrm{ABF}$ was statistically related with $\mathrm{BFR}$.

The reason for making holes in the flap is to perform central tack-up sutures known as "Poppen's sutures." Central tack-up sutures are important for the prevention of postoperative extradural hematomas..$^{20}$ In general, holes are made using a thin burr, such as a wire-pass drill. However, passing the surgical thread through a narrow hole is usually troublesome. Thus, some neurosurgeons have started to make holes using thick (5-mm) burrs, and this was found to be significantly associated with BFR in this study (Fig. 5).

The mechanism by which wide holes promote BFR is not clear. We assumed that this phenomenon was related to cancellous bone. The skull consists of internal and external tables or layers of cortical bone separated by cancellous bone..$^{21}$ Making a hole in the $\mathrm{ABF}$ reduces the cortical bone and exposes the cancellous bone to osteoclasts circulating in the blood, which may have promoted bone resorption. The cancellous bone of the parietal bone is also exposed along the craniotomy margin, and indeed, 
we noted a large bony gap at the parietal area because the $\mathrm{ABF}$ was tightly attached to the frontal bone. Thus, the cancellous bone of the parietal bone was largely exposed to blood. The frontal bone also contains cancellous bone. However, the frontal bone was less exposed to blood because it was closely attached to the calvaria. Therefore, BFR was less frequent, and sometimes bone union occurred at the frontal area.

This study had some limitations. First, it was relatively small and employed a retrospective design. Second, follow-up CT periods were not consistent. Hence, a large and prospective study will be required in the future.

Most ABFs after cranioplasty are absorbed, and severe BFR with less than $60 \%$ of the initial bone flap volume is relatively frequent, often leading to repeated cranioplasty and increasing the overall medical cost, the possibility of a medical dispute, morbidity, and mortality. These shortcomings should be fully explained to patients who are about to undergo cranioplasty with $\mathrm{ABF}$. Furthermore, making wide holes in ABFs should be avoided to reduce the risk for BFR, and in cases that central tack-up sutures are required, narrow holes are recommended.

\section{ACKNOWLEDGEMENTS}

This work was supported by the Medical ICT Convergence Center of the Busan Economic Promotion Agency.

\section{AUTHOR CONTRIBUTIONS}

Conceptualization: Chung Kee Chough. Data curation: Hyuk Jin Choi, Won Ho Cho, Seung Heon Cha, Chang Hwa Choi, Young Ha Kim. Formal analysis: Hyuk Jin Choi. Funding acquisition: Jung Hwan Lee. Investigation: Jung Hwan Lee. Methodology: Jung Hwan Lee, Chung Kee Chough. Project administration: Chung Kee Chough. Resources: Jung Hwan Lee. Software: Jung Hwan Lee. Supervision: Chung Kee Chough. Validation: Jun Kyeung Ko, Young Ha Kim. Visualization: Jung Hwan Lee. Writing_original draft: Jung Hwan Lee. Writing_review \& editing: Chung Kee Chough, Won Ho Cho.

\section{ORCID iDs}

Jung Hwan Lee https://orcid.org/0000-0002-1393-7105 Chung Kee Chough https://orcid.org/0000-0001-7810-8992 Hyuk Jin Choi https://orcid.org/0000-0002-7314-2267 Jun Kyeung Ko https://orcid.org/0000-0002-5652-7659 Won Ho Cho https://orcid.org/0000-0003-2058-1554 Seung Heon Cha https://orcid.org/0000-0002-0570-1477 Chang Hwa Choi https://orcid.org/0000-0001-6430-1396 Young Ha Kim https://orcid.org/0000-0002-6790-8799

\section{REFERENCES}

1. Hofmeijer J, Kappelle LJ, Algra A, Amelink GJ, van Gijn J, van der Worp HB; HAMLET investigators. Surgical decompression for spaceoccupying cerebral infarction (the Hemicraniectomy After Middle Cerebral Artery infarction with Life-threatening Edema Trial [HAMLET]): a multicentre, open, randomised trial. Lancet Neurol
2009;8:326-33.

2. Fisher CM, Ojemann RG. Bilateral decompressive craniectomy for worsening coma in acute subarachnoid hemorrhage. Observations in support of the procedure. Surg Neurol 1994;41:65-74.

3. Guerra WK, Gaab MR, Dietz H, Mueller JU, Piek J, Fritsch MJ. Surgical decompression for traumatic brain swelling: indications and results. J Neurosurg 1999;90:187-96.

4. Honeybul S, Ho KM. How "successful" is calvarial reconstruction using frozen autologous bone? Plast Reconstr Surg 2012;130:1110-7.

5. Dujovny M, Fernandez P, Alperin N, Betz W, Misra M, Mafee M. Post-cranioplasty cerebrospinal fluid hydrodynamic changes: magnetic resonance imaging quantitative analysis. Neurol Res 1997; 19:311-6.

6. Winkler PA, Stummer W, Linke R, Krishnan KG, Tatsch K. Influence of cranioplasty on postural blood flow regulation, cerebrovascular reserve capacity, and cerebral glucose metabolism. J Neurosurg 2000;93:53-61.

7. Honeybul S. Decompressive craniectomy: a new complication. J Clin Neurosci 2009;16:727-9.

8. Iwama T, Yamada J, Imai S, Shinoda J, Funakoshi T, Sakai N. The use of frozen autogenous bone flaps in delayed cranioplasty revisited. Neurosurgery 2003;52:591-6.

9. Kim JS, Cheong JH, Ryu JI, Kim JM, Kim CH. Bone flap resorption following cranioplasty after decompressive craniectomy: preliminary report. Korean J Neurotrauma 2015;11:1-5.

10. Park SP, Kim JH, Kang HI, Kim DR, Moon BG, Kim JS. Bone flap resorption following cranioplasty with autologous bone: quantitative measurement of bone flap resorption and predictive factors. J Korean Neurosurg Soc 2017;60:749-54.

11. Grant GA, Jolley M, Ellenbogen RG, Roberts TS, Gruss JR, Loeser JD. Failure of autologous bone-assisted cranioplasty following decompressive craniectomy in children and adolescents. J Neurosurg 2004;100(2 Suppl Pediatrics):163-8.

12. Lee JH, Han IH, Choi BK, Nam KH, Kim DH, Lee CS. Virtual preoperative simulation for excision of spinal tumors: surgeon processing of medical computer-assisted design software. Korean J Spine 2017;14:170-4.

13. Vanaclocha V, Sáiz-Sapena N, García-Casasola C, De Alava E. Cranioplasty with autogenous autoclaved calvarial bone flap in the cases of tumoural invasion. Acta Neurochir (Wien) 1997;139:970-6.

14. Lee SH, Yoo CJ, Lee U, Park CW, Lee SG, Kim WK. Resorption of autogenous bone graft in cranioplasty: resorption and reintegration failure. Korean J Neurotrauma 2014;10:10-4.

15. Osawa M, Hara H, Ichinose Y, Koyama T, Kobayashi S, Sugita Y. Cranioplasty with a frozen and autoclaved bone flap. Acta Neurochir (Wien) 1990;102:38-41.

16. Prolo DJ, Oklund SA. Composite autogeneic human cranioplasty: frozen skull supplemented with fresh iliac corticocancellous bone. Neurosurgery 1984;15:846-51.

17. Dünisch P, Walter J, Sakr Y, Kalff R, Waschke A, Ewald C. Risk factors of aseptic bone resorption: a study after autologous bone flap reinsertion due to decompressive craniotomy. J Neurosurg 2013; 118:1141-7.

18. Brommeland T, Rydning PN, Pripp AH, Helseth E. Cranioplasty complications and risk factors associated with bone flap resorption. Scand J Trauma Resusc Emerg Med 2015;23:75.

19. Schwarz F, Dünisch P, Walter J, Sakr Y, Kalff R, Ewald C. Cranioplasty after decompressive craniectomy: is there a rationale for an initial artificial bone-substitute implant? A single-center experience after 631 procedures. J Neurosurg 2016;124:710-5.

20. Sharma M, Madhugiri V, Nanda A. James L. Poppen and surgery of the "seat of the soul": a contemporary perspective. World Neurosurg 2014;82:529-34.

21. Nair AP, Mehrotra A, Das KK, Kumar B, Srivastav AK, Sahu RN, et al. Calvarial tuberculosis of the parietal bone: a rare complication after dental extraction. Asian J Neurosurg 2015;10:219-21. 